\title{
Influence of Water Quality, Formulation, Adjuvant, Rainfastness, and Nozzle Type on Efficacy of Fomesafen on Palmer Amaranth (Amaranthus palmeri) Control
}

\author{
Vijay K. Nandula1 ${ }^{*}$, William T. Molin ${ }^{1}$, Jason A. Bond ${ }^{2}$ \\ ${ }^{1}$ United States Department of Agriculture, Agricultural Research Service, Stoneville, MS, USA \\ ${ }^{2}$ Delta Research and Extension Center, Mississippi State University, Stoneville, MS, USA \\ Email: ${ }^{\star}$ vijay.nandula@ars.usda.gov
}

How to cite this paper: Nandula, V.K., Molin, W.T. and Bond, J.A. (2018) Influence of Water Quality, Formulation, Adjuvant, Rainfastness, and Nozzle Type on Efficacy of Fomesafen on Palmer Amaranth (Amaranthus palmeri) Control. American Journal of Plant Sciences, 9, 1660-1676. https://doi.org/10.4236/ajps.2018.98120

Received: June 23, 2018

Accepted: July 16, 2018

Published: July 19, 2018

Copyright $\odot 2018$ by authors and Scientific Research Publishing Inc. This work is licensed under the Creative Commons Attribution International License (CC BY 4.0).

http://creativecommons.org/licenses/by/4.0/

(c) (i) Open Access

\begin{abstract}
Protoporphyrinogen oxidase (PPO) inhibitors are one of the few remaining postemergence herbicide options for controlling Palmer amaranth in soybean growing areas of Mississippi, USA. Most Palmer amaranth populations in Mississippi are resistant to both glyphosate and acetolactate synthase inhibitors. Resistance to PPO inhibiting herbicides in Palmer amaranth has very recently been reported in Arkansas, Tennessee, and isolated pockets of Mississippi. A significant proportion of reports of PPO inhibitor failures in Mississippi are not considered to be resistance-related at this time. Therefore, the objective of this research was to evaluate factors affecting the efficacy of fomesafen on Palmer amaranth including: quality of spray carrier (water), formulations, adjuvant, rainfastness, and nozzle type. All water samples and formulation combinations provided $>95 \%$ control of Palmer amaranth 3 WAT. Some combinations of water samples and formulations did not result in complete control of the treated plants, with one or two individuals surviving 3 WAT. Formulation 1 provided 99\% control compared to 95\% from formulation 2. Irrespective of combinations of herbicide, adjuvant and height, control of Palmer amaranth was $\geq 91 \%$. Formulation 1 provided $94 \%$ control compared to $88 \%$ from formulation 2 . The adjuvant $\mathrm{x}$ height interaction was significant, owing to a $10 \%$ reduction in control of larger plants $(86 \%)$ compared to smaller plants (96\%) in presence of COC. COC provided better control (93\%) than NIS (88\%). Simulated rainfall applied $\geq 60 \mathrm{~min}$ after herbicide application did not adversely affect efficacy on Palmer amaranth when formulation 1 was applied in combination with NIS, with control ranging from $94 \%$ to $100 \%$. Formulation 1 with COC provided $\geq 93 \%$ control at all rainfall
\end{abstract}


application times, except 30 min after herbicide treatment, which resulted in $79 \%$ control. Formulation 2 provided better control with COC (79\% to $100 \%$ ) than NIS (71\% to $90 \%)$, in general, across the rainfall treatments applied at various times following herbicide application. All nozzle and weed height combinations resulted in $89 \%$ or better control of Palmer amaranth. In summary, water quality, formulation, adjuvant, rainfastness, or nozzle type did not affect the activity of fomesafen under optimal application conditions in the greenhouse.

\section{Keywords}

Adjuvant, Fomesafen, Formulation, Nozzle, Palmer Amaranth, Rainfastness, Soybean, Water Quality

\section{Introduction}

Widespread distribution of glyphosate-resistant (GR) weeds in soybean [ Glycine $\max ($ L.) Merr.]-growing areas across Mississippi has economically affected soybean planting and follow-up crop management operations. Several of the GR weeds, especially pigweeds (Amaranthus spp.), are also resistant to acetolactate synthase (ALS)-inhibiting herbicides [1] thereby, limiting the number of herbicide choices for post weed control. Protoporphyrinogen oxidase (PPO) inhibitors are one of the few remaining postemergence (POST) weed control herbicide options, another being glufosinate in LibertyLink ${ }^{\circledast}$ (glufosinate-resistant) soybean, for soybean growers in Mississippi, USA.

New multiple herbicide-resistant crop technologies, involving dicamba and 2,4-D resistance, with associated formulations have been deregulated (transgenic traits by USDA)/registered (herbicide formulations by EPA), but their performance on large production fields is not clear. In 2016, several growers across the states of Arkansas, Missouri, Tennessee, and to a lesser extent in Mississippi treated fields planted to dicamba-resistant cotton (Gossypium hirsutum L.) and soybean with unauthorized/off-label/unregistered dicamba formulations, thereby, injuring large swaths of non dicamba-resistant soybean fields in the midsouthern US [2]. Injury to soybean from dicamba applications took a new turn in 2017. Labeled applications of registered dicamba formulations on dicamba-resistant soybean drifted (volatile/vapor drift and/or physical drift due to droplet movement owing to temperature inversion and other factors) off-target and injured an alarmingly large area, 1 million ha across the midwestern and southern U.S. [3] and 1.5 million ha across the U.S.

(https://ipm.missouri.edu/IPCM/2017/10/final_report_dicamba_injured_soybea

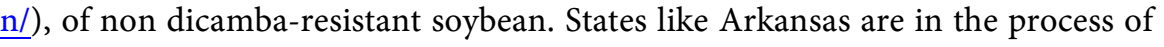
legislatively limiting the window of application of dicamba on dicamba-resistant soybean in the 2018 growing season [4]. Similar issues will most likely be encountered with 2,4-D-resistant crop technologies when commercialized. In addition, effectiveness of these technologies could be short-lived as it has been 
shown that Palmer amaranth can become less susceptible under conditions of continuous exposure to sub-lethal doses of dicamba [5].

Resistance to PPO-inhibiting herbicides in Palmer amaranth [Amaranthus palmeri (S.) Wats.] has recently been documented in Arkansas and Tennessee [6]. To date, resistance to PPO inhibitors in Mississippi has been isolated [7]. Recent research reported less than acceptable levels of control of Palmer amaranth from PPO inhibitors such as fomesafen [7], which indicates a developing issue of resistance to PPO inhibitors in Mississippi. It is not clear if field failures of PPO inhibitors are due to resistance or misapplication/adverse application conditions. Under the uncertain conditions of the utility of auxin $(2,4-\mathrm{D}$ and dicamba)-resistant crop technologies and the potential for wide spread development of PPO-resistant weed populations in Mississippi, prolonging the sustainability of PPO herbicides for MS soybean producers is of paramount importance.

Adjuvants improve an herbicide's efficacy [8] [9] by increasing its absorption [10] [11]. Adequate absorption across the leaf cuticle is key to the performance of contact type herbicides such as PPO inhibitors. The performance of adjuvants is influenced by the herbicide with which they are used, the weed species, water quality, and prevailing weather conditions [8] [10] [12]. Rainfastness is the property of an herbicide to be effective, via adequate drying on the applied plant or absorption by plant tissues, before the first rain after application. It can influence effectiveness of herbicides, particularly, contact type.

Therefore, the objective of this research was to evaluate the effect of water quality, formulation, adjuvant, rainfastness, and nozzle type on efficacy of fomesafen, a PPO inhibitor, on susceptible/wild type Palmer amaranth.

\section{Materials and Methods}

\subsection{Seeding, Plant Growth, and Herbicide Treatment Conditions}

All experiments were conducted in a greenhouse at the Jamie Whitten Delta States Research Center of USDA-ARS in Stoneville, Mississippi set to $25 / 20^{\circ} \mathrm{C} \pm$ $3^{\circ} \mathrm{C}$ day/night temperature under ambient conditions. Wild type/susceptible (to all major families of herbicides, data not shown) Palmer amaranth seed was sown at a depth of $0.5 \mathrm{~cm}$ in plastic trays $(50 \mathrm{~cm} \times 20 \mathrm{~cm} \times 6 \mathrm{~cm})$ containing a commercial potting mix [formulated Canadian sphagnum peat moss, coarse perlite, bark ash, starter nutrient charge (with gypsum) and slow release nitrogen and dolomitic limestone] (Metro-Mix 360, Sun Gro Horticulture, Bellevue, WA) and watered. Two weeks after germination, $2.5-\mathrm{cm}$ tall seedlings were transplanted into $8 \mathrm{~cm} \times 8 \mathrm{~cm} \times 7 \mathrm{~cm}$ pots containing the same potting mix. Thereafter, plants were watered as needed and fertilized once two weeks after transplanting with a water-soluble fertilizer (Miracle-Gro, Scotts Miracle-Gro Products, Inc., Marysville, OH). All herbicide treatment were applied using an air-pressurized indoor spray chamber (DeVries Manufacturing Co., Hollandale, MN) equipped with a nozzle mounted with 8002E flat-fan tip (Spraying Systems Co., Wheaton, IL), except additional nozzles being included in the Nozzle study, 
delivering $190 \mathrm{~L} \cdot \mathrm{ha}^{-1}$ at $220 \mathrm{kPa}$. Fomesafen was applied at $0.42 \mathrm{~kg} \cdot \mathrm{ai} \cdot \mathrm{ha}^{-1}$, single highest dose recommended in Mississippi [13], in all experiments. All herbicide treatments were evaluated for efficacy based on percent control ratings $(0=$ no injury, $100=$ dead) recorded $3 \mathrm{wk}$ after treatment $($ WAT). Percent mortality was recorded 3 WAT only in the Water Quality and Formulation study. A plant was considered a survivor when there was evidence of green tissue in the axillary and/or terminal growing points. An individual plant represented one replication. There were 10 replications per treatment in the Water Quality and Formulation study, and 4 replications per treatments in all the other studies.

\subsection{Water Quality and Formulation}

All water samples were collected in 2016 in clean 3.8-L plastic containers and stored at 2 to $8 \mathrm{C}$ until further use. Water sources included city or well at mixing facilities of participating members (commercial applicators) of the Mississippi Agricultural Aviation Association, county agents, and industry representatives (Table 1, Figure 1). Detailed information on water sources was provided only by few cooperators. Sources of water samples collected from the same county were at least $25 \mathrm{~km}$ apart to maintain randomness and uniqueness of location. Global Positioning System (GPS) coordinates were available for only a few of the samples, hence not shown. These water sources draw from deep groundwater aquifers, opposed to shallow groundwater aquifers which are primarily used for irrigation purposes. Aircraft applicators made up a bulk of the chosen sources since they apply herbicides on the largest crop area based on unit water source. An aliquot of each water sample was analyzed for selected properties by a commercial agricultural analytical laboratory (Waypoint Analytical, Memphis, TN, USA). A representative analytical report is shown in Figure 2. Palmer amaranth plants, 5- to $10-\mathrm{cm}$ tall, three to six true leaves, were treated with three formulations: Flexstar ${ }^{\oplus}$ (formulation 1, 22.1\% a.i., Syngenta Crop Protection, Greensboro, NC), Reflex (formulation 2, 22.8\% a.i., Syngenta Crop Protection), and Top Gun $^{\oplus}$ (formulation 3, 22.8\% a.i., Loveland Products, Inc., Greeley, CO), all at $0.42 \mathrm{~kg}$ ai/ha, using city or well water samples as spray carrier. All treatments included had crop oil concentrate (COC, Agridex ${ }^{\oplus}$, Helena Chemical Co., Collierville, $\mathrm{TN}$ ) at $1 \% \mathrm{v} / \mathrm{v}$.

\subsection{Formulation and Adjuvant}

Both formulations 1 (Flexstar $\left.{ }^{\circledast}\right)$ and $2\left(\right.$ Reflex $\left.^{\circledast}\right)$, described previously, were applied with a nonionic surfactant (NIS, Induce ${ }^{\oplus}$, Helena Chemical Co.) at $0.25 \%$ $\mathrm{v} / \mathrm{v}$ and a COC at $1 \% \mathrm{v} / \mathrm{v}$ to plants at four different growth stages, 2.9 to $3.8 \mathrm{~cm}$, 5.6 to $7 \mathrm{~cm}, 9.1$ to $9.6 \mathrm{~cm}$, and 11.6 to $13.5 \mathrm{~cm}$.

\subsection{Adjuvant Rate}

Both formulations 1 (Flexstar $\left.{ }^{\oplus}\right)$ and $2\left(\right.$ Reflex $\left.^{\oplus}\right)$ were applied with an NIS at 0.25 and $0.5 \% \mathrm{v} / \mathrm{v}$ and a COC at 1 and $2 \% \mathrm{v} / \mathrm{v}$ to plants at two different growth stages, 11.5 to 15.5 and 24.8 to $26.8 \mathrm{~cm}$. 
Table 1. Details of water sampling locations and summary of water quality analyses. ${ }^{\mathrm{a}}$

\begin{tabular}{|c|c|c|c|c|c|c|c|c|c|}
\hline Sample\# & County & Source & $\mathrm{pH}$ & Hardness & $\mathrm{Fe}$ & $\mathrm{CO}_{3}$ & $\mathrm{HCO}_{3}$ & $\mathrm{Na}$ & $\mathrm{Cl}$ \\
\hline & & & & \multicolumn{6}{|c|}{$\mathrm{mg} \cdot \mathrm{L}^{-1}$} \\
\hline 1 & Bolivar & City & 8.3 & 2.09 & 0.06 & 22 & 333 & 171 & 45 \\
\hline 2 & Bolivar & City & 8.3 & 8.08 & 0.36 & 24 & 478 & 418 & 242 \\
\hline 3 & Bolivar & Well & 8.1 & 449 & 0.8 & 39 & 384 & 18 & 37 \\
\hline 4 & Bolivar & City & 8.4 & 18.2 & 0.35 & 36 & 323 & 166 & 39 \\
\hline 5 & Bolivar & & 8.7 & 1.33 & 0.05 & 39 & 101 & 100 & 30 \\
\hline 6 & Bolivar & City & 8.5 & 4.46 & 0.05 & 29 & 483 & 231 & 35 \\
\hline 7 & Bolivar & City & 8.6 & 2.07 & 0.06 & 34 & 434 & 189 & 20 \\
\hline 8 & Bolivar & City & 8.3 & 22.4 & 0.05 & 39 & 338 & 163 & 37 \\
\hline 9 & Coahoma & Well & 8.0 & 277 & 0.53 & 39 & 197 & 12 & 42 \\
\hline 10 & DeSoto & Well & 7.7 & 266 & 13.7 & 0 & 278 & 14 & 14 \\
\hline 11 & DeSoto & City & 8.0 & 12.2 & 0.16 & 10 & 145 & 65 & 11 \\
\hline 12 & Humphreys & & 8.2 & 5.34 & 0.09 & 24 & 163 & 78 & 18 \\
\hline 13 & Humphreys & & 8.3 & 1.95 & 0.05 & 22 & 163 & 90 & 12 \\
\hline 14 & Issaquena & & 8.6 & 3.06 & 0.05 & 49 & 483 & 282 & 81 \\
\hline 15 & Issaquena & & 8.4 & 3.04 & 0.05 & 29 & 471 & 281 & 75 \\
\hline 16 & Leflore & & 8.0 & 240 & 1.48 & 32 & 249 & 11 & 17 \\
\hline 17 & Leflore & & 8.4 & 3.89 & 0.05 & 49 & 259 & 136 & 7 \\
\hline 18 & Leflore & & 8.4 & 12.6 & 0.06 & 44 & 293 & 138 & 12 \\
\hline 19 & Leflore & & 8.5 & 3.98 & 0.05 & 39 & 269 & 126 & 12 \\
\hline 20 & Leflore & & 8.5 & 2.73 & 0.05 & 19 & 259 & 127 & 7 \\
\hline 21 & Madison & & 8.2 & 6.6 & 0.05 & 39 & 212 & 172 & 41 \\
\hline 22 & Sharkey & Well & 7.9 & 419 & 2.22 & 27 & 419 & 15 & 11 \\
\hline 23 & Sharkey & & 9.0 & 1.78 & 0.05 & 87 & 392 & 223 & 30 \\
\hline 24 & Sharkey & & 8.7 & 2.43 & 0.05 & 36 & 394 & 201 & 33 \\
\hline 25 & Sharkey & & 8.5 & 2.42 & 0.05 & 44 & 382 & 199 & 36 \\
\hline 26 & Tallahatchie & City & 8.4 & 8.81 & 0.05 & 10 & 328 & 408 & 289 \\
\hline 27 & Tallahatchie & City & 8.3 & 3.81 & 0.12 & 24 & 274 & 147 & 27 \\
\hline 28 & Tallahatchie & & 7.9 & 18.4 & 0.05 & 19 & 163 & 72 & 21 \\
\hline 29 & Tallahatchie & & 8.5 & 4.37 & 0.14 & 51 & 234 & 121 & 28 \\
\hline 30 & Tallahatchie & & 8.0 & 7.23 & 0.2 & 27 & 269 & 148 & 41 \\
\hline 31 & Washington & & 8.1 & 76.5 & 0.05 & 32 & 338 & 116 & 22 \\
\hline 32 & Washington & Well & 8.7 & 2.61 & 0.05 & 61 & 407 & 231 & 20 \\
\hline 33 & Washington & & 8.7 & 2.56 & 0.07 & 63 & 490 & 314 & 100 \\
\hline 34 & Washington & & 8.0 & 384 & 1.21 & 36 & 421 & 47 & 30 \\
\hline 35 & Washington & & 8.5 & 6.39 & 0.05 & 58 & 333 & 177 & 30 \\
\hline 36 & Washington & & 8.0 & 76 & 0.68 & 34 & 446 & 226 & 65 \\
\hline 37 & Washington & City & 8.4 & 3.09 & 0.05 & 32 & 224 & 151 & 38 \\
\hline 38 & Washington & Well & 9.1 & 2.36 & 0.05 & 95 & 352 & 241 & 81 \\
\hline 39 & & Distilled Water & 6.1 & 1.05 & 0.05 & 0 & 10 & 0 & 5 \\
\hline
\end{tabular}

${ }^{\mathrm{a}}$ The levels for each of the water quality parameters indicating severe, slight to moderate, and no problems/issues, respectively, were established as follows: $\mathrm{pH}$ : $>7.9,<5.8$ and 7.1 - 7.9, $5.8-7$; hardness: $>180,60$ - 180, <60; Fe: >1.5, $0.3-1.5,<0.3 ; \mathrm{CO}_{3}:>510,120-510,<120 ; \mathrm{HCO}_{3}:>519,122-519,<122$; Na: >138, $69-$ 138, <69; Cl: >179, $107-179,<107$. 


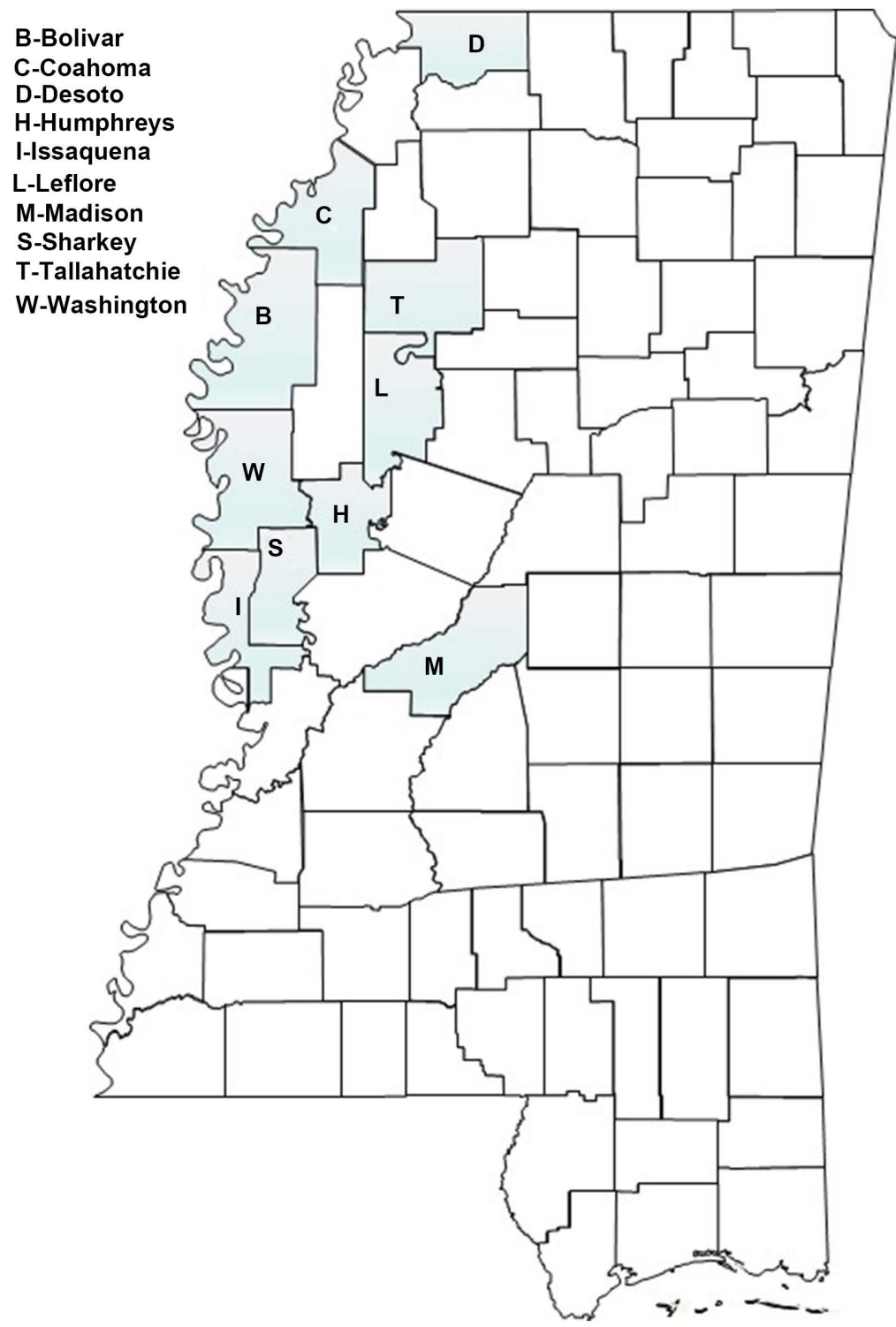

Figure 1. Map of counties in Mississippi where water samples were collected.

\subsection{Rainfastness}

Both formulations 1 (Flexstar ${ }^{\circledR}$ ) and $2\left(\right.$ Reflex $\left.^{\oplus}\right)$ were applied with an NIS at $0.25 \% \mathrm{v} / \mathrm{v}$ and a COC at $1 \% \mathrm{v} / \mathrm{v}$ to $10-\mathrm{cm}$-tall plants. Treated plants were sprayed with simulated rainfall equivalent to $0.5 \mathrm{~cm}$ [14] for a duration of $0,10,30,60$, 120 , and $240 \mathrm{~min}$. After each rainfall timing, plants were returned to the greenhouse.

\subsection{Nozzle}

In this experiment, nine different nozzles, 8002, Airmix 110-02 (agrotop, Obertraubling, Germany), TT360, AITT36011002, AI11002VS, TTI02, DG11002VS, AIXR11002, 11002, were evaluated using the indoor spray chamber mentioned before. All nozzles, except Airmix 11-02, were acquired from Spraying Systems Co., Wheaton, IL, USA. Formulation $2\left(\right.$ Reflex $\left.^{\otimes}\right)$ was applied with a COC at $1 \%$ 


\section{Waypoint}

2790 Whitten Road, Memphis, TN 38133

Main $901.213 .2400^{\circ}$ Fax 901.213.2440 www.waypointanalytical.com

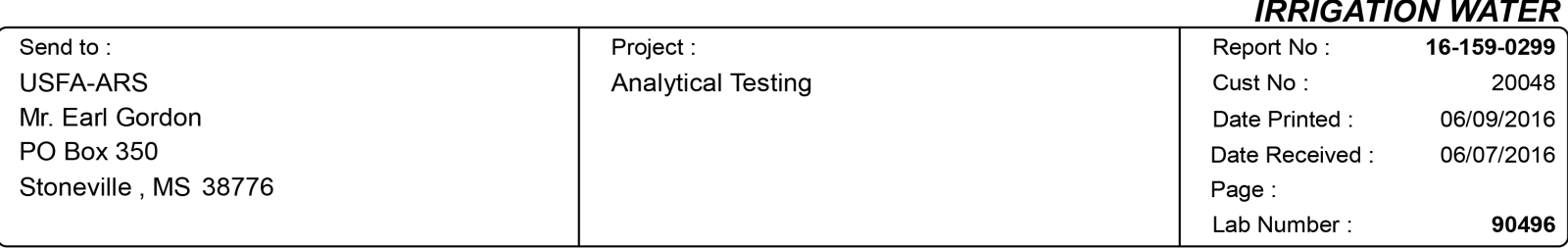

Sample Id : 1

\begin{tabular}{|c|c|c|c|}
\hline \multicolumn{2}{|l|}{ CATIONS } & $\overline{\mathrm{mg} / \mathrm{L}}$ & $\overline{\mathrm{meq} / \mathrm{L}}$ \\
\hline \multicolumn{2}{|l|}{ Sodium } & 14 & 0.61 \\
\hline \multicolumn{2}{|l|}{ Calcium } & 77 & 3.84 \\
\hline \multicolumn{2}{|l|}{ Magnesium } & 18 & 1.48 \\
\hline \multicolumn{2}{|l|}{ Potassium } & 3 & 0.08 \\
\hline \multirow{2}{*}{ Ammonium } & $\mathrm{NH}_{4}$ & 1 & 0.07 \\
\hline & $\mathrm{NH}_{4}-\mathrm{N}$ & 1 & \\
\hline \multicolumn{3}{|c|}{ SUM OF CATIONS } & 6.08 \\
\hline
\end{tabular}

\begin{tabular}{|l|c|c|c|}
\hline \multicolumn{2}{|l|}{ ANIONS } & $\mathrm{mg} / \mathrm{L}$ & $\mathrm{meq} / \mathrm{L}$ \\
\hline \multirow{2}{*}{ Chloride } & $\mathrm{Cl}$ & 14 & 0.39 \\
\hline \multirow{2}{*}{ Sulfate } & $\mathrm{SO}_{4}$ & 21 & 0.44 \\
\cline { 2 - 4 } & $\mathrm{S}$ & 7 & \\
\hline Bicarbonate & $\mathrm{HCO}_{3}$ & 278 & 4.56 \\
\hline Carbonate & $\mathrm{CO}_{3}$ & 0 & 0.00 \\
\hline \multirow{2}{*}{ Nitrate } & $\mathrm{NO}_{3}$ & 0 & 0.00 \\
\cline { 2 - 4 } & $\mathrm{NO}_{3}-\mathrm{N}$ & 0 & 0.06 \\
\hline \multirow{2}{*}{ Phosphate } & $\mathrm{PO}_{4}$ & 2 & \\
\cline { 2 - 5 } & $\mathrm{P}^{*}$ & 1 & \\
\hline \multirow{2}{*}{ sum OF ANIONS } & &
\end{tabular}

\begin{tabular}{|c|c|c|c|}
\hline Hydrogen Ion Activity & $\mathrm{pH}$ & 7.7 & \\
\hline Equilibrium Reaction & $\mathrm{pHc}$ & 6.17 & \\
\hline Electrical Conductivity & ECw & 0.53 & $\mathrm{dS} / \mathrm{m}$ \\
\hline Total Dissolved Solids & TDS & 339 & $\mathrm{mg} / \mathrm{L}$ \\
\hline Adj $\mathrm{Na}$ Adsorption Ratio & SARadj & 0.48 & \\
\hline Sodium Adsorption Ratio & SAR & 0.37 & \\
\hline Hardness & & 266 & ppm \\
\hline
\end{tabular}

\begin{tabular}{|ll|c|}
\hline Copper & $\mathrm{Cu}$ & $0.01 \mathrm{mg} / \mathrm{L}$ \\
\hline Zinc & $\mathrm{Zn}$ & $0.06 \mathrm{mg} / \mathrm{L}$ \\
\hline Manganese & $\mathrm{Mn}$ & $0.85 \mathrm{mg} / \mathrm{L}$ \\
\hline Iron & $\mathrm{Fe}$ & $13.70 \mathrm{mg} / \mathrm{L}$ \\
\hline Boron & $\mathrm{B}$ & $0.05 \mathrm{mg} / \mathrm{L}$ \\
\hline Fluoride & $\mathrm{F}$ & \\
\hline Aluminum & $\mathrm{Al}$ & $0.40 \mathrm{mg} / \mathrm{L}$ \\
\hline Molybdenum & $\mathrm{Mo}$ & $0.02 \mathrm{mg} / \mathrm{L}$ \\
\hline
\end{tabular}

$\mathrm{mg} / \mathrm{L}=$ parts per million parts water

meq/L - milliequivalents per liter

Hardness is determined from calculations using the calcium and magnesium concentrations in the water.

TDS calculated by ECW * 640

DISCLAIMER: The following water analysis interpretation should serve only as a guideline. It should not be used without considering crop type, soil chemistry, plant growth environment and water management practices. Consult a local or state soil and water specialist for a more thorough evaluation of your water's quality.

\section{Page 2 of 51}

(a) 


\section{Waypoint $^{\infty}$}

2790 Whitten Road, Memphis, TN 38133

Main 901.213.2400 ${ }^{\circ}$ Fax 901.213.2440 www.waypointanalytical.com

\begin{tabular}{|c|c|c|c|}
\hline Send to: & Project & Report No: & $16-159-0299$ \\
\hline USFA-ARS & Analytical Testing & Cust No : & 20048 \\
\hline Mr. Earl Gordon & & Date Printed: & 06/09/2016 \\
\hline PO Box 350 & & Date Received & $06 / 07 / 2016$ \\
\hline \multirow[t]{2}{*}{ Stoneville, MS 38776} & & Page: & \\
\hline & & Lab Number: & 90496 \\
\hline
\end{tabular}

Sample Id : 1

WATER ANALYSIS INTERPRETATION, AGRICULTURAL

\begin{tabular}{|c|c|c|c|c|c|c|c|c|}
\hline \multirow{3}{*}{ Potential Problem } & \multirow{3}{*}{ Units } & \multirow{3}{*}{$\begin{array}{c}\text { Test } \\
\text { Result }\end{array}$} & \multicolumn{6}{|c|}{ Degree of Restriction on Use } \\
\hline & & & \multicolumn{3}{|c|}{ Criteria } & \multicolumn{3}{|c|}{ Graphical Results } \\
\hline & & & None & Slight to Moderate & Severe & None & Slight to Moderate & Severe \\
\hline Salinity & & & & & & & & \\
\hline $\mathrm{ECw}^{1}$ & $\mathrm{dS} / \mathrm{m}$ & 0.53 & $<0.7$ & $0.7-3$ & $>3$ & 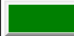 & & \\
\hline Specific Ion Toxicity & & & & & & & & \\
\hline Sodium $(\mathrm{Na})^{1}$ & & & & & & & & \\
\hline Surface irrigation & SARadj & 0.48 & $<3$ & $3-9$ & $>9$ & D & & \\
\hline Sprinkler irrigation ${ }^{2}$ & $\mathrm{meq} / \mathrm{L}$ & 0.61 & $<3$ & $3-6$ & $>6$ & 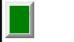 & & \\
\hline Chloride $(\mathrm{Cl})^{1}$ & & & & & & & & \\
\hline Surface irrigation & $\mathrm{meq} / \mathrm{L}$ & 0.39 & $<4$ & $4-10$ & $>10$ & 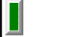 & & \\
\hline Sprinkler irrigation ${ }^{2}$ & $\mathrm{meq} / \mathrm{L}$ & 0.39 & $<3$ & $3-5$ & $>5$ & D & & \\
\hline Boron (B) ${ }^{1}$ & $\mathrm{mg} / \mathrm{L}$ & 0.05 & $<0.7$ & $0.7-3$ & $>3$ & 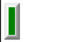 & & \\
\hline Fluoride $(F)^{1}$ & & & $<1$ & $1-5$ & $>5$ & & & \\
\hline Clogging of Drip Systems or Unsightly Residues & & & & & & & & \\
\hline Iron $(\mathbf{F e})^{3}$ & $\mathrm{mg} / \mathrm{L}$ & 13.70 & $<0.3$ & $0.3-1.5$ & $>1.5$ & D & & \\
\hline Manganese $(M n)^{3}$ & $\mathrm{mg} / \mathrm{L}$ & 0.85 & $<0.2$ & $0.2-1.5$ & $>1.5$ & & & \\
\hline $\mathrm{pH}-\mathrm{pHc}^{4}$ & & 1.53 & $<=0$ & $>0$ & & & & \\
\hline $\begin{array}{l}\text { Reduced Water Infiltration } \\
\text { ( Ratio based on adjSAR / ECW ) }\end{array}$ & & 0.91 & $<4$ & $4-10$ & $>10$ & 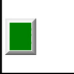 & & \\
\hline Alkalinity & & & & & & & & \\
\hline Bicarbonate $\left(\mathrm{HCO}_{3}\right)+$ Carbonate $\left(\mathrm{CO}_{3}\right)^{6}$ & meq/L & 4.56 & $<2$ & $2-8.5$ & $>8.5$ & & & \\
\hline Potential Low Nutrient Issues (Soilless media) & & & & & & & & \\
\hline Sulfate & $\mathrm{mg} / \mathrm{L}$ & 21 & $>48$ & $48-20$ & $<20$ & & & \\
\hline Magnesium & $\mathrm{mg} / \mathrm{L}$ & 18 & $>10$ & $10-4$ & $<4$ & 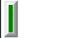 & & \\
\hline Boron & $\mathrm{mg} / \mathrm{L}$ & 0.05 & $>0.3$ & $0.3-0.05$ & $<0.05$ & & & \\
\hline
\end{tabular}

1. Crop tolerance to salinity, sodium, chloride, boron and fluoride varies widely. Most tree crops are sensitive to sodium and chloride while many annual crops are not. Soil conditions, irrigation method and climate must be considered.

2. Leaf burn from foliar and root absorption will be enhanced under conditions of : low humidity, high temperature and high air movement .

3. Elevated iron in combination with sulfides or tannins can result in bacterial slimes that can clog drip systems. Removal of iron and manganese often involves oxidation ( aeration or chlorination ) followed by filtering.

4. Positive $\mathrm{pH}-\mathrm{pHc}$ ( saturation index ) values indicate the potential for calcium and magnesium carbonate precipitates that might impair efficiency of irrigation systems with small orificed parts and/or may leave unsightly lime deposits on leaves. Problems can be reduced by mineral acid addition.

5. Infiltration problems are most likely when water with low ECW and/or high SAR adj. is used on mineral soils containing some silt and clay.

Evaluation of infiltration problems should include analysis of both irrigation water and soil-water extracts. Treatment may involve injecting gypsum into the water or applying gypsum to the soil surface.

6. Bicarbonate when excessive may result in difficulty in controlling soil pH and may impair root assimilation of minor elements.

7. Sulfur, magnesium and /or boron may become limiting if not supplied by soil or fertilizer. Use soil and leaf analysis to confirm need.

Comments : 


\section{Waypoint}

2790 Whitten Road, Memphis, TN 38133

Main $901.213 .2400^{\circ}$ Fax 901.213.2440 www.waypointanalytical.com

IRRIGATION WATER

\begin{tabular}{|c|c|c|c|}
\hline Send to & Project & Report No: & $16-159-0299$ \\
\hline USFA-ARS & Analytical Testing & Cust No: & 20048 \\
\hline Mr. Earl Gordon & & Date Printed : & 06/09/2016 \\
\hline PO Box 350 & & Date Received & $06 / 07 / 2016$ \\
\hline \multirow[t]{2}{*}{ Stoneville, MS 38776} & & Page & \\
\hline & & Lab Number: & 90496 \\
\hline
\end{tabular}

Sample Id : 1

SPRAY WATER ANALYSIS INTERPRETATION

\begin{tabular}{|l|c|c|c|c|c|c|}
\hline Potential Problem & pH & Hardness & Iron & Carbonate & Bicarbonate & Sodium \\
\hline Test Result & 7.7 & 266 & 13.70 & 0 & 278 & $\begin{array}{c}14 \\
\mathrm{mg} / \mathrm{L}\end{array}$ \\
Units & $\mathrm{s.u}$ & $\mathrm{mg} / \mathrm{L}$ & $\mathrm{mg} / \mathrm{L}$ & $\begin{array}{c}14 \\
\mathrm{mg} / \mathrm{L}\end{array}$ \\
\hline
\end{tabular}

\begin{tabular}{|l|c|c|c|c|c|c|}
\hline Severe & $>7.9$ & $>180$ & $>1.5$ & $>510$ & $>519$ & $>138$ \\
\hline Slight to Moderate & $<5.8 ; 7.1-7.9$ & $60-180$ & $0.3-1.5$ & $120-510$ & $122-519$ & $69-138$ \\
\hline None & $5.8-7$ & $<60$ & $<0.3$ & $<120$ & $<122<179$ \\
\hline
\end{tabular}

\begin{tabular}{|c|c|c|c|c|c|c|}
\hline \multicolumn{7}{|c|}{ Severe } \\
\hline \multicolumn{7}{|c|}{ Moderate } \\
\hline \multicolumn{7}{|c|}{ Slight } \\
\hline None & & & & & 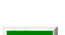 & \\
\hline & Hardness & $\mathrm{Fe}$ & $\mathrm{CO}_{3}$ & $\mathrm{HCO}_{3}$ & $\mathrm{Na}$ & $\mathrm{Cl}$ \\
\hline
\end{tabular}

One or more potential problems are moderate to severe. Consider the use of a water conditioner or a different water source. Water Hardness indicates water conditioning recommended.

For weak acid herbicides (which includes most of those applied post), buffering is recommended when $\mathrm{pH}$ exceeds 7.5 . Optimum range for $\mathrm{pH}$ is between 3.0 and 6.0 .

For insecticide/fungicide active ingredients that are subject to decomposition by alkaline hydrolysis, buffer addition is recommended when $\mathrm{pH}$ exceeds 7.0. Optimum range is $\mathrm{pH}$ between 3.0 and 5.0 depending on active ingredient.

For glyphosate, buffering is recommended when $\mathrm{pH}$ exceeds 5.0. Optimum range is $\mathrm{pH}$ between 3.0 and 4.5 .

Iron at this level may antagonize glyphosate.

Figure 2. A representative water analysis report. of counties in Mississippi where water samples were collected. 
$\mathrm{v} / \mathrm{v}$ to plants at 3 growth stages, 4.25 to $6,6.13$ to 8 , and 10.4 to $13.8 \mathrm{~cm}$. formulations 1 (Flexstar $\left.{ }^{\circledast}\right)$ and $2\left(\right.$ Reflex $\left.^{\circledast}\right)$ were applied with an NIS at 0.25 and $0.5 \%$ $\mathrm{v} / \mathrm{v}$ and a COC at 1 and $2 \% \mathrm{v} / \mathrm{v}$ to plants at two different growth stages, 11.5 to 15.5 and 24.8 to $26.8 \mathrm{~cm}$.

\subsection{Statistical Analyses}

All experiments were conducted using a completely randomized design and repeated. Data from all experiments were subjected to ANOVA using the PROC GLM statement in SAS (version 9.2, SAS Institute, Inc., Cary, NC, USA). Data from repeated experiments were pooled due to a non-significant experimental effect. Treatment means were separated using Fisher's Protected LSD test at P = 0.05 .

\section{Results and Discussion}

\subsection{Water Quality and Formulation}

Analytical report for each water sample included individual estimates of cations such as $\mathrm{Na}^{+}, \mathrm{Ca}^{2+}, \mathrm{Mg}^{2+}, \mathrm{K}^{+}$, and $\mathrm{NH}_{4}^{+}$, anions such as $\mathrm{Cl}^{-}, \mathrm{SO}_{4}^{2-}, \mathrm{S}^{2-}, \mathrm{HCO}_{3}^{-}$, $\mathrm{CO}_{3}^{2-}, \mathrm{NO}_{3}^{-}, \mathrm{PO}_{4}^{3-}$, and $\mathrm{P}^{3-}$, minerals such as $\mathrm{Cu}, \mathrm{Zn}, \mathrm{Mn}, \mathrm{Fe}, \mathrm{B}, \mathrm{F}, \mathrm{Al}$, and Mo, and other parameters such as $\mathrm{pH}$, electrical conductivity, and hardness (Figure 2). The levels for each of the water quality parameters indicating severe, slight to moderate, and no problems/issues, respectively, were established as follows: $\mathrm{pH}:>7.9,<5.8$ and 7.1 - 7.9, 5.8 - 7; hardness: >180, $60-180,<60$; Fe: $>1.5$, 0.3 - 1.5, <0.3; $\mathrm{CO}_{3}:>510,120$ - 510, <120; $\mathrm{HCO}_{3}:>519,122$ - 519, <122; Na: >138, 69 - 138, <69; Cl: >179, 107 - 179, <107 (Table 1). Herbicide applicators will, no doubt, add buffering and conditioning agents to the water before large-scale treatment of fields. However, we added no amendments to the water samples before testing for efficacy of fomesafen on Palmer amaranth. All results including analytical reports and efficacy results have been shared with cooperating aircraft applicators, county agents and growers.

There was no impact of water quality, formulation or water quality $\mathrm{x}$ formulation interaction on Palmer amaranth control and mortality (data not shown). All water samples and formulation combinations provided $>95 \%$ control of Palmer amaranth 3 WAT (data not shown). Some combinations of water samples and formulations did not result in complete control of the treated plants, with one or two individuals surviving 3 WAT (Table 2). Overall, water quality did not adversely affect the efficacy of any of the three fomesafen formulations evaluated despite the marked variation (Table 1 ) observed in the levels of various parameters measured across different locations.

In Tables 3-6, treatment means for significant main and interaction effects are provided followed by p-values for all sources included in the ANOVA model. The last set of values in any given table represent treatment means of all factors in the interaction containing all main effects, except where such an interaction was significant and whose values have already been reported in the table prior to the $\mathrm{p}$-values. 
Table 2. Effect of water quality and fomesafen formulation on Palmer amaranth mortality, 3 wk after treatment.

\begin{tabular}{|c|c|c|}
\hline Fomesafen & Sample\# & Mortality \\
\hline & & $\%$ \\
\hline \multirow[t]{3}{*}{ Formulation 1} & 1 & 90 \\
\hline & 2 & 95 \\
\hline & 4 & 90 \\
\hline \multirow[t]{9}{*}{ Formulation 2} & 1 & 95 \\
\hline & 2 & 95 \\
\hline & 3 & 90 \\
\hline & 4 & 90 \\
\hline & 5 & 95 \\
\hline & 7 & 95 \\
\hline & 31 & 95 \\
\hline & 37 & 95 \\
\hline & 39 & 95 \\
\hline \multirow[t]{7}{*}{ Formulation 3} & 1 & 95 \\
\hline & 4 & 95 \\
\hline & 6 & 95 \\
\hline & 7 & 95 \\
\hline & 36 & 95 \\
\hline & 38 & 95 \\
\hline & 39 & 95 \\
\hline
\end{tabular}

\subsection{Formulation and Adjuvant}

Among main and interaction effects, only the formulation main effect impacted control of Palmer amaranth (Table 3). Formulation 1 provided 99\% control compared to $95 \%$ from formulation 2. Irrespective of combinations of herbicide, adjuvant and height, control of Palmer amaranth was $\geq 91 \%$ (Table 3 ).

\subsection{Adjuvant Rate}

Among main effects, formulation impacted control of Palmer amaranth (Table 4). Formulation 1 provided $94 \%$ control compared to $88 \%$ from formulation 2. The adjuvant $\mathrm{x}$ height interaction was significant, owing to a $10 \%$ reduction in control of larger plants (86\%) compared to smaller plants (96\%) in presence of COC (Table 4).

\subsection{Rainfastness}

Adjuvant type had a significant impact on Palmer amaranth control (Table 5). COC provided better control (93\%) than NIS (88\%). The three-way interaction 
Table 3. Effect of fomesafen formulation and adjuvant on Palmer amaranth control, 3 wk after treatment. ${ }^{\mathrm{a}}$

\begin{tabular}{|c|c|c|c|c|}
\hline Main/Interaction factor & & & $P$ value & Control \\
\hline & & & & $\%$ \\
\hline Formulation 1 & & & & 99 \\
\hline Formulation 2 & & & & 95 \\
\hline $\operatorname{LSD}(0.05)$ & & & & 3 \\
\hline Formulation & & & 0.0116 & \\
\hline Adjuvant & & & 0.9391 & \\
\hline Height & & & 0.1927 & \\
\hline Formulation $\times$ adjuvant & & & 0.7599 & \\
\hline Formulation $\times$ height & & & 0.5037 & \\
\hline Adjuvant $\times$ height & & & 0.6252 & \\
\hline Formulation $\times$ adjuvant $\times$ height & & & 0.9470 & \\
\hline \multicolumn{5}{|c|}{ Main factor } \\
\hline \multirow[t]{8}{*}{ Formulation 1} & NIS & Height 1 & & 100 \\
\hline & & Height 2 & & 100 \\
\hline & & Height 3 & & 100 \\
\hline & & Height 4 & & 98 \\
\hline & $\mathrm{COC}$ & Height 1 & & 100 \\
\hline & & Height 2 & & 96 \\
\hline & & Height 3 & & 99 \\
\hline & & Height 4 & & 100 \\
\hline \multirow[t]{8}{*}{ Formulation 2} & NIS & Height 1 & & 100 \\
\hline & & Height 2 & & 94 \\
\hline & & Height 3 & & 91 \\
\hline & & Height 4 & & 93 \\
\hline & $\mathrm{COC}$ & Height 1 & & 100 \\
\hline & & Height 2 & & 91 \\
\hline & & Height 3 & & 94 \\
\hline & & Height 4 & & 95 \\
\hline
\end{tabular}

${ }^{a}$ Abbreviations: COC, crop oil concentrate; NIS nonionic surfactant.

between formulation adjuvant and rainfall timing after herbicide treatment was significant for Palmer amaranth control. Simulated rainfall applied $\geq 60$ min after herbicide application did not adversely affect efficacy on Palmer amaranth when formulation 1 was applied in combination with NIS, with control ranging from $94 \%$ to $100 \%$. Formulation 1 with COC provided $\geq 93 \%$ control at all rainfall application times, except $30 \mathrm{~min}$ after herbicide treatment, which resulted in 79\% control. Formulation 2 provided better control with COC (79\% to 100\%) than NIS ( $71 \%$ to $90 \%)$, in general, across the rainfall treatments applied at various times following herbicide application. 
Table 4. Effect of fomesafen formulation, adjuvant and adjuvant rate on Palmer amaranth control, 3 wk after treatment. ${ }^{\text {a }}$

\begin{tabular}{|c|c|c|c|c|c|}
\hline Main/Interaction factor & & & & $P$ value & Control \\
\hline & & & & & $\%$ \\
\hline Formulation 1 & & & & & 94 \\
\hline Formulation 2 & & & & & 88 \\
\hline \multirow[t]{5}{*}{$\operatorname{LSD}(0.05)$} & & & & & 5 \\
\hline & NIS & & Height 1 & & 88 \\
\hline & & & Height 2 & & 93 \\
\hline & $\mathrm{COC}$ & & Height 1 & & 97 \\
\hline & & & Height 2 & & 86 \\
\hline $\operatorname{LSD}(0.05)$ & & & & & 7 \\
\hline Formulation & & & & 0.0111 & \\
\hline Adjuvant & & & & 0.6864 & \\
\hline Adjuvant rate & & & & 0.1658 & \\
\hline Height & & & & 0.2182 & \\
\hline Formulation $\times$ adjuvant & & & & 0.6180 & \\
\hline Formulation $\times$ adjuvant rate & & & & 0.4449 & \\
\hline Formulation $\times$ height & & & & 0.8866 & \\
\hline Adjuvant $\times$ height & & & & 0.0049 & \\
\hline Formulation $\times$ adjuvant $\times$ adjuvant rate & & & & 0.1822 & \\
\hline Formulation $\times$ adjuvant $\times$ height & & & & 0.0548 & \\
\hline \multicolumn{3}{|l|}{ Formulation $\times$ adjuvant $\times$ adjuvant rate $\times$ height } & & 0.1281 & \\
\hline \multicolumn{6}{|c|}{ Main factor } \\
\hline \multirow[t]{8}{*}{ Formulation 1} & NIS & 0.25 & Height 1 & & 94 \\
\hline & & & Height 2 & & 97 \\
\hline & & 0.5 & Height 1 & & 95 \\
\hline & & & Height 2 & & 92 \\
\hline & $\mathrm{COC}$ & 1 & Height 1 & & 96 \\
\hline & & & Height 2 & & 96 \\
\hline & & 2 & Height 1 & & 98 \\
\hline & & & Height 2 & & 88 \\
\hline \multirow[t]{8}{*}{ Formulation 2} & NIS & 0.25 & Height 1 & & 87 \\
\hline & & & Height 2 & & 84 \\
\hline & & 0.5 & Height 1 & & 77 \\
\hline & & & Height 2 & & 98 \\
\hline & $\mathrm{COC}$ & 1 & Height 1 & & 100 \\
\hline & & & Height 2 & & 89 \\
\hline & & 2 & Height 1 & & 94 \\
\hline & & & Height 2 & & 73 \\
\hline
\end{tabular}

${ }^{a}$ Abbreviations: COC, crop oil concentrate; NIS nonionic surfactant.

\subsection{Nozzle}

Neither of the main effects, nozzle type nor height of Palmer amaranth, nor the interaction significantly influenced Palmer amaranth control when treated with 
Table 5. Effect of rainfastness on efficacy of fomesafen on Palmer amaranth, 3 wk after treatment. ${ }^{\mathrm{a}}$

\begin{tabular}{|c|c|c|c|c|}
\hline Main/Interaction factor & & & $P$ value & Control \\
\hline & & & & $\%$ \\
\hline NIS & & & & 93 \\
\hline $\mathrm{COC}$ & & & & 88 \\
\hline $\operatorname{LSD}(0.05)$ & & & & 5 \\
\hline \multirow[t]{12}{*}{ Formulation 1} & NIS & MAT 0 & & 86 \\
\hline & & MAT 10 & & 85 \\
\hline & & MAT 30 & & 85 \\
\hline & & MAT 60 & & 100 \\
\hline & & MAT 120 & & 94 \\
\hline & & MAT 240 & & 100 \\
\hline & $\mathrm{COC}$ & MAT 0 & & 100 \\
\hline & & MAT 10 & & 95 \\
\hline & & MAT 30 & & 79 \\
\hline & & MAT 60 & & 100 \\
\hline & & MAT 120 & & 98 \\
\hline & & MAT 240 & & 93 \\
\hline \multirow[t]{12}{*}{ Formulation 2} & NIS & MAT 0 & & 90 \\
\hline & & MAT 10 & & 89 \\
\hline & & MAT 30 & & 89 \\
\hline & & MAT 60 & & 83 \\
\hline & & MAT 120 & & 88 \\
\hline & & MAT 240 & & 71 \\
\hline & $\mathrm{COC}$ & MAT 0 & & 79 \\
\hline & & MAT 10 & & 90 \\
\hline & & MAT 30 & & 87 \\
\hline & & MAT 60 & & 100 \\
\hline & & MAT 120 & & 96 \\
\hline & & MAT 240 & & 100 \\
\hline LSD (0.05) & & & & 3 \\
\hline Formulation & & & 0.0556 & \\
\hline Adjuvant & & & 0.0407 & \\
\hline MAT & & & 0.1159 & \\
\hline Formulation $\times$ adjuvant & & & 0.3040 & \\
\hline Formulation $\times$ MAT & & & 0.2892 & \\
\hline Adjuvant $\times$ MAT & & & 0.4768 & \\
\hline Formulation $\times$ adjuvant $\times$ MAT & & & 0.0063 & \\
\hline
\end{tabular}

aAbbreviations: COC, crop oil concentrate; MAT, min after treatment; NIS nonionic surfactant. 
Table 6. Effect of nozzle type on efficacy of fomesafen on Palmer amaranth, 3 wk after treatment.

\begin{tabular}{|c|c|c|c|}
\hline Main/Interaction factor & & $P$ value & Control \\
\hline & & & $\%$ \\
\hline Nozzle type & & 0.3755 & \\
\hline Height & & 0.2051 & \\
\hline Nozzle type $\times$ Height & & 0.9204 & \\
\hline \multirow[t]{3}{*}{8002} & Height 1 & & 100 \\
\hline & Height 2 & & 95 \\
\hline & Height 3 & & 100 \\
\hline \multirow[t]{3}{*}{ Airmix 110-02 } & Height 1 & & 100 \\
\hline & Height 2 & & 100 \\
\hline & Height 3 & & 100 \\
\hline \multirow[t]{3}{*}{ TT360 } & Height 1 & & 100 \\
\hline & Height 2 & & 100 \\
\hline & Height 3 & & 95 \\
\hline \multirow[t]{3}{*}{ AITT36011002 } & Height 1 & & 100 \\
\hline & Height 2 & & 98 \\
\hline & Height 3 & & 90 \\
\hline \multirow[t]{3}{*}{ AI11002V } & Height 1 & & 94 \\
\hline & Height 2 & & 100 \\
\hline & Height 3 & & 89 \\
\hline \multirow[t]{3}{*}{ TTI02 } & Height 1 & & 94 \\
\hline & Height 2 & & 100 \\
\hline & Height 3 & & 89 \\
\hline \multirow[t]{3}{*}{ DG11002VS } & Height 1 & & 100 \\
\hline & Height 2 & & 100 \\
\hline & Height 3 & & 100 \\
\hline \multirow[t]{3}{*}{ AIXR11002 } & Height 1 & & 100 \\
\hline & Height 2 & & 100 \\
\hline & Height 3 & & 100 \\
\hline \multirow[t]{3}{*}{11002} & Height 1 & & 100 \\
\hline & Height 2 & & 100 \\
\hline & Height 3 & & 100 \\
\hline
\end{tabular}

formulation 2 in combination with COC (Table 6). All nozzle and weed height combinations resulted in $89 \%$ or better control of Palmer amaranth (Table 6).

In summary, water quality, formulation, adjuvant, rainfastness, or nozzle type did not affect efficacy of fomesafen on Palmer amaranth. Of course, activity of other PPO inhibitors could be influenced by one or more of the above parameters. Reports of PPO inhibitor failures in the field must be taken seriously after considering the role of these factors on each individual reported case. 


\section{Conclusions}

This research, especially, with water quality analysis, is an "out-of-the-box" approach to eliminate factors that may result in the mis-diagnosis of non-performance of a PPO inhibitor as a case of resistance. An aircraft applicator from DeSoto County, Mississippi has indicated his readiness to switch from a city water supply to a well at his mixing facility to save on costs, based on results on water quality provided to him from this research.

Uncertainties in commercialization of auxin-resistant crop technologies, coupled with related escalating rhetoric and lack of postemergence herbicide alternatives in soybean with no major herbicide mode of action commercialized in about 20 years [15], have severely limited weed management strategies for soybean growers of Mississippi. Additionally, sporadic development of resistance to PPO inhibiting herbicides in pigweed populations of Mississippi has been documented and there exists a potential for the spread of resistance on a broader scale due to repeated selection pressure and other non-mitigating factors. Hence, the research reported here is very relevant in that application parameters evaluated here can be modified to prolong the sustainability of PPO inhibitors as well as slow the spread of resistance to PPO inhibitors such as fomesafen, which is clearly the preferred treatment in glyphosate-resistant soybean. The research described above can be applicable to several post emergence applied herbicides such as other PPO inhibitors (e.g., acifluorfen, lactofen, saflufenacil), ALS inhibitors (e.g., chlorimuron, imazamox, pyrithiobac, penoxsulam), ACCase inhibitors (e.g., fenoxaprop, clethodim, pinoxaden), photosystem II inhibitors (e.g., atrazine), photosystem I inhibitors (ex. paraquat), glyphosate, glufosinate, 2,4-D, and dicamba labeled for use in various crops including soybean, corn, and cotton grown in the southeastern US.

Fomesafen can be an effective weed management tool for growers in Mississippi and other parts of the US and the world, provided, other control strategies such as cultural and mechanical practices are incorporated in to an integrated weed management program. The results from this research indicate that while some Palmer amaranth populations exhibit trending resistance to fomesafen, fomesafen and other PPO inhibitors still have a role in broad leaf weed management programs. Nevertheless, non performance of all herbicides should be evaluated by taking both biotic (weed growth stage, etc.) and abiotic (weather, application parameters such as water quality, formulation, adjuvant, rainfastness, nozzles, etc.) factors into account.

\section{Disclaimer}

Mention of trade names or commercial products in this publication is solely for the purpose of providing specific information and does not imply recommendation or endorsement by the US Department of Agriculture.

\section{Acknowledgements}

The authors are grateful for funding from the Mississippi Soybean Promotion 
Board. The authors thank Collin Rounsavall and Chris Suddoth for their assistance in performing these experiments.

\section{References}

[1] Nandula, V.K., Reddy, K.N., Koger, C.H., Poston, D.H., Rimando, A.M., Duke, S.O., Bond, J.A. and Ribeiro, D.N. (2012) Multiple Resistance to Glyphosate and Pyrithiobac in Palmer Amaranth (Amaranthus palmeri) from Mississippi and Response to Flumiclorac. Weed Science, 60, 179-188. https://doi.org/10.1614/WS-D-11-00157.1

[2] Delta Farm Press (2018). http://www.deltafarmpress.com/soybeans/improper-dicamba-use-leaves-mid-south -multitude-drift-cases

[3] Cornucopia (2018). https://www.cornucopia.org/2017/08/dicamba-drift-damaged-2-5-million-acres-co unting/

[4] Reuters (2018). https://www.reuters.com/article/us-usa-pesticides-arkansas/arkansas-may-bar-dica mba-herbicide-use-after-april-15-idUSKCN1B52OM

[5] Tehranchian, P., Norsworthy, J.K., Powles, S., Bararpour, M.T., Bagavathiannan, M.V., Barber, T. and Scott, R.C. (2017) Recurrent Sublethal-Dose Selection for Reduced Susceptibility of Palmer Amaranth (Amaranthus palmeri) to Dicamba. Weed Science, 65, 206-212. https://doi.org/10.1017/wsc.2016.27

[6] Delta Farm Press (2018). http://www.deltafarmpress.com/weed-control/ppo-resistant-pigweeds-confirmed-ar kansas-tennessee

[7] Nandula, V.K. (2017) An Update on Mississippi State-Wide Herbicide Resistance Screening in Pigweed (Amaranthus) Populations. Proceedings of the Weed Science Society of America, Tucson, AZ; Weed Science Society of America, Westminster, CO, 219.

[8] Hatzios, K.K. and Penner, D. (1985) Interactions of Herbicides with Other Agrichemicals in Higher Plants. Reviews of Weed Science, 1, 1-63.

[9] Wanamarta, G. and Penner, D. (1989) Identification of Efficacious Adjuvants for Sethoxydim and Bentazon. Weed Technology, 3, 60-66. https://doi.org/10.1017/S0890037X00031328

[10] Hull, H.M., Davis, G.D. and Stoltenberg G.E. (1982) Action of Adjuvants on Plant Surfaces. In: Hodgson, R.H., Ed., Adjuvants for Herbicides, Weed Science Society of America, Champaign, IL, 26-67.

[11] Wanamarta, G. and Penner, D. (1989) Foliar Penetration of Herbicides. Reviews of Weed Science, 4, 215-231.

[12] McWhorter, C.G. (1982) The Use of Adjuvants. In: Hodgson, R.H., Ed., Adjuvants for Herbicides, Weed Science Society of America, Champaign, IL, 11-25.

[13] Mississippi State University (2017) Weed Control Guidelines for Mississippi. Mississippi State University, Mississippi.

[14] Reddy, K.N. and Locke, M.A. (1996) Imazaquin Spray Retention, Foliar Washoff and Runoff Losses under Simulated Rainfall. Pesticide Science, 48, 179-187. https://doi.org/10.1002/(SICI)1096-9063(199610)48:2<179::AID-PS457>3.0.CO;2-M

[15] Duke, S.O. (2012) Why Have No New Modes of Action Appeared in Recent Years? Pest Management Science, 68, 505-512. https://doi.org/10.1002/ps.2333 